\title{
An Admissible Semantics for Propositionally Quantified Relevant Logics
}

\author{
Robert Goldblatt and Michael Kane \\ Centre for Logic, Language and Computation \\ Victoria University of Wellington, New Zealand
}

3 June 2009

\begin{abstract}
The Routley-Meyer relational semantics for relevant logics is extended to give a sound and complete model theory for many propositionally quantified relevant logics (and some non-relevant ones). This involves a restriction on which sets of worlds are admissible as propositions, and an interpretation of propositional quantification that makes $\forall p A$ true when there is some true admissible proposition that entails all $p$-instantiations of $A$.

It is also shown that without the admissibility qualification many of the systems considered are semantically incomplete, including all those that are sub-logics of the quantified version of Anderson and Belnap's system E of entailment, extended by the mingle axiom and the Ackermann constant $t$. The incompleteness proof involves an algebraic semantics based on atomless complete Boolean algebras.
\end{abstract}

\section{Introduction}

Propositional quantification played a role in the early development of ideas about relevant implication. Anderson and Belnap observed in [2] that enriching their entailment system $\mathrm{E}$ by quantifiers $\forall p, \exists p$ binding propositional variables allowed the definition of other conditionals. Thus an enthymematic conditional $A \supset B$, i.e. one with a suppressed true assumption, could be defined as

$$
\exists p(p \wedge(A \wedge p \rightarrow B)),
$$

where $\rightarrow$ is the implication of system E. Strict implication $A \rightarrow B$ could be defined as

$$
\exists p(N p \wedge(A \wedge p \rightarrow B)),
$$

where $N$ is the necessity modality defined by taking $N p$ to be $(p \rightarrow p) \rightarrow p$. They stated that adding the quantifiers to the positive fragment of $E$ gives a system whose theorems in $-3, \wedge$ and $\vee$ coincide exactly with the positive fragment of Lewis's system S4 of strict implication, and whose theorems in $\supset, \wedge$ and $\vee$ coincide exactly with the positive fragment of Heyting's system of intuitionistic logic. Also, if the negation $\neg A$ is defined as $A \supset(\forall p) p$, then the theorems in $\supset, \neg, \wedge$ and $\vee$ coincide exactly with the full intuitionistic propositional calculus. Here $(\forall p) p$ serves as the Falsum, an absurdity implying every proposition.

No axioms for the quantifiers were stated in [2], but these were supplied by Anderson in [1], extending $\mathrm{E}$ and the system $\mathrm{R}$ of relevant implication to logics $\mathrm{E} \dagger$ and $\mathrm{R} \dagger$ whose 
quantifier axioms were the universal closures of the schemes

$$
\begin{aligned}
& \forall p A \rightarrow A[B / p] \\
& \forall p(A \rightarrow B) \rightarrow(\forall p A \rightarrow \forall p B) \\
& \forall p A \wedge \forall p B \rightarrow \forall p(A \wedge B) \\
& \forall p(A \rightarrow B) \rightarrow(A \rightarrow \forall p B), \text { with } p \text { not free in } A . \\
& \forall p(A \vee B) \rightarrow(A \vee \forall p B), \text { with } p \text { not free in } A . \\
& (\forall p(p \rightarrow p) \rightarrow A) \rightarrow A .
\end{aligned}
$$

Meyer in [9] gave alternative axiomatisations of these logics, calling them EP and RP. He studied the above conditional definitions and others, verifying the assertions about connections with intuitionistic logic and S4. He also noted that the Ackermann constant $t$, thought of as the conjunction of all truths, could be quantificationally defined as $\forall p(p \rightarrow p)$.

The volume [3] devoted its first chapter to relevant systems extended by propositional quantification, using the notation (which we adopt) $S^{\forall p}$ for some system $S$ thus extended.

A semantics for propositional quantifiers was discussed by Routley and Meyer when they introduced their possible-worlds style model theory for the logic R in [10]. Their model structures carry a quasi-order $\leq$, and propositions are interpreted to be subsets of the structure that are hereditary, i.e. closed upward under the quasi-order, as in Kripke's intuitionistic semantics. They observed that taking $\forall$ to mean "for all hereditary subsets" gives a sound semantics - all theorems of RP are validated - but stated their belief that completeness fails. This was by analogy with Henkin's primary interpretations of higher-order logic [6], given that this interpretation of $\forall$ was second-order in nature. Kremer [7] eventually proved their conjecture by showing that the set of formulas validated by the Routley-Meyer primary semantics for RP is not recursively axiomatisable.

The present paper provides a complete relational semantics for RP, EP and other propositionally quantified relevant logics. The initial idea is to restrict the class of hereditary sets that are admissible as propositions. Each model structure will have a fixed collection Prop of hereditary sets over which the propositional variables range. We require Prop to be closed under the operations interpreting the logical connectives. This approach has been successfully used to model non-quantified (Boolean) propositional modal logics that are incomplete for their Kripke semantics, and has also been applied to some substructural logics $^{1}$. But here we have the new question of how to interpret the propositional quantifiers relative to Prop.

Our answer, in brief, is an old one from algebraic logic: a universal quantifier is interpreted by a greatest lower bound in the lattice of propositions, this being the natural interpretation of arbitrary conjunctions. An approach of this kind was developed for quantification of individual variables in [8]. Here it is adapted to quantification of propositional variables. To explain how this works, let $\forall p A$ be a sentence, and $A(P)$ be the result of replacing free $p$ in $A$ by the hereditary set (proposition) $P$, viewed as a constant. Let $|\forall p A|$ and $|A(P)|$ be the hereditary sets of worlds at which these sentences are true, respectively. The RoutleyMeyer primary semantics in effect takes $\forall p A$ to have the same meaning as the conjunction of the $A(P)^{\prime}$ 's as $P$ ranges over all hereditary sets, so puts

$$
|\forall p A|=\bigcap\{|A(P)|: P \text { is hereditary }\} .
$$

This makes $|\forall p A|$ the greatest lower bound of the $|A(P)|$ 's in the set of all hereditary sets under the partial order $\subseteq$ of set inclusion. That partial order is also the interpretation of the entailment relation between propositions.

In a model whose set Prop of admissible propositions contains only some of the hereditary sets, we take

$$
|\forall x A|=\prod_{P \in \text { Prop }}|A(P)|
$$

\footnotetext{
${ }^{1}$ For instance in [4], where admissible propositions for an Action Logic related to dynamic logic are taken to be certain "stable" subsets of a canonical model. That paper does not discuss propositional quantification.
} 
where $\Pi$ denotes greatest lower bound in the ordered set $($ Prop,$\subseteq)$. Our definition of "model" will require that $\prod_{P \in \text { Prop }}|A(P)|$ always exists in Prop. But it may not be equal to the intersection $\bigcap\{|A(P)|: P \in P r o p\}$. Instead it will be the largest admissible proposition included in this intersection, and hence the union of all admissible propositions included in the intersection. Writing $a \models \forall p A$ for " $\forall p A$ is true at world $a$ ", i.e. $a \in|\forall x A|$, we get that

$$
a \models \forall p A \text { iff there is some } X \in \operatorname{Prop} \text { such that } a \in X \text { and } X \subseteq \bigcap_{P \in P \text { rop }}|A(P)| \text {. }
$$

Thus

$\forall p A$ is true at $a$ iff some admissible proposition true at $a$ entails every admissible instantiation $A(P)$ of $A$.

Our "old" use of greatest lower bounds as conjunctions provides a new semantic propositional analysis of the meaning of $\forall p$.

To develop a semantics that can interpret all formulas and not just sentences, we need to assign propositions to variables. A formula $A$ with $n$ free variables $p_{1}, \ldots, p_{n}$ can be seen as defining an $n$-ary propositional function, i.e. a function of the form Prop $^{n} \rightarrow$ Prop, taking each $n$-tuple $P_{1}, \ldots, P_{n}$ of admissible propositions to the proposition $\left|A\left(P_{1}, \ldots, P_{n}\right)\right|$ expressed by $A$ when each $p_{i}$ is assigned the value $P_{i}$. Since different formulas may have different numbers of free variables, this approach would involve handling finitary propositional functions of different arities, which would quickly become cumbersome. A more convenient and equally natural approach is to use functions of the form Prop ${ }^{\omega} \rightarrow$ Prop, where $\omega=\{0,1,2, \ldots\}$. An element $f \in \operatorname{Prop}^{\omega}$ is a function $f: \omega \rightarrow \operatorname{Prop}$ that serves as a valuation assigning the proposition $f(n)$ to the variable $p_{n}$ for all $n \in \omega$, and so is a device that gives a value to all variables simultaneously. Such an $f$ can be thought as a sequence $f(0), \ldots, f(n), \ldots$ of admissible propositions. Each formula $A$ determines a propositional function $|A|:$ Prop $^{\omega} \rightarrow$ Prop, taking each $f \in$ Prop $^{\omega}$ to the admissible proposition $|A| f$ expressed by $A$ when its free variables are interpreted according to $f .|A|$ is defined formally by induction on the length of $A$, as will be seen in Section 3.

Now just as we do not admit arbitrary hereditary sets as propositions, so too we do not expect an arbitrary function from Prop ${ }^{\omega}$ to Prop to be the interpretation of a logical formula. In addition to Prop, our model structures have a fixed collection PropF un of admissible propositional functions that is closed under function-building operations interpreting the connectives and quantifiers. These closure properties ensure that $|A| \in$ PropFun for any formula $A$, and hence that $|A| f$ is always admissible.

As well as proving soundness and completeness of many logics under our semantics, we also give incompleteness results showing that our admissible-propositions approach is essential. These results demonstrate that many of our logics are incomplete for validity in models in which every hereditary set is admissible. This is done by exhibiting a particular sentence that is valid in all such models but not a theorem of the logic in question. The latter part of the proof requires the development of an algebraic semantics using Boolean algebras that are order-complete but atomless.

The next section defines the many logics we study and gives their pertinent prooftheoretic properties. Section 3 defines our model structures and models, and gives the soundness theorem for the weakest logic. Section 4 proves the completeness theorem for this logic by a canonical model construction, and then Section 5 extends these results to all the other logics. The final Section 6 gives the incompleteness results via algebraic semantics.

\section{Logics}

Our formal language is based on a countably infinite set $\operatorname{Var}=\left\{p_{0}, p_{1}, p_{2}, \ldots\right\}$ of propositional variables, and a countably infinite set $C$ on of propositional constants (we will usually use the letter $c$, possibly with subscripts, to refer to members of $C o n$ ). Formulas are generated from these variables and constants in the standard way, using the connectives $\rightarrow, \wedge, \neg$; a special propositional constant $t$; and the universal quantifiers $\forall p_{n}$. We also employ the 
abbreviations

$$
\begin{array}{rlrl}
A \vee B & =_{d f} & \neg(\neg A \wedge \neg B) \\
A \leftrightarrow B & =_{d f} & & (A \rightarrow B) \wedge(B \rightarrow A) \\
\exists p A & ={ }_{d f} & \neg \forall p(\neg A) .
\end{array}
$$

The notions of free and bound occurrences of variables and of $p_{n}$ being free for $B$ in $A$, are as usual. A formula is closed, or is a sentence, if it has no free variables. To deal with substitution of formulas for variables the notation

$$
A\left[B_{0} / p_{0}, \ldots, B_{n} / p_{n}, \ldots\right]
$$

will refer to the formula resulting from simultaneous substitution of each $B_{i}$ for all free occurrences of $p_{i}$ in $A$. A single substitution $A\left[p_{0} / p_{0}, \ldots, B / p_{i}, \ldots, p_{n} / p_{n}, \ldots\right]$ will be abbreviated to $A\left[B / p_{i}\right]$, and similarly we define any finite substitution $A\left[B_{0} / p_{n_{0}}, \ldots, B_{m} / p_{n_{m}}\right]$ in the obvious way.

\section{Axiom Schemes:}

A1. $\quad A \rightarrow A$

A2. $\quad A \wedge B \rightarrow A$

A3. $\quad A \wedge B \rightarrow B$

A4. $\quad(A \rightarrow B) \wedge(A \rightarrow C) \rightarrow(A \rightarrow B \wedge C)$

A5. $\quad A \rightarrow A \vee B$

A6. $\quad B \rightarrow A \vee B$

A7. $\quad(A \rightarrow C) \wedge(B \rightarrow C) \rightarrow(A \vee B \rightarrow C)$

A8. $\quad A \wedge(B \vee C) \rightarrow(A \wedge B) \vee(A \wedge C)$

A9. $\neg \neg A \rightarrow A$

A10. $\forall p_{n} A \rightarrow A\left[B / p_{n}\right] \quad$ (where $p_{n}$ is free for $B$ in $A$ )

Rules: 2

(R1)

$$
\frac{A \rightarrow B}{A}
$$

$$
\frac{A \rightarrow B}{(B \rightarrow C) \rightarrow(A \rightarrow C)}
$$

(R5) $\frac{A \rightarrow \neg B}{B \rightarrow \neg A}$

(R7) $\frac{A}{t \rightarrow A}$

$$
\begin{gathered}
A \\
B \\
\hline A \wedge B
\end{gathered}
$$

$$
\frac{B \rightarrow C}{(A \rightarrow B) \rightarrow(A \rightarrow C)}
$$

(R6) $\frac{t \rightarrow A}{A}$

(RIC) $\frac{A \rightarrow B}{A \rightarrow \forall p_{n} B}$ if $p_{n}$ is not free in $A$

By a logic we mean any set $L$ of formulas that includes all instances of these axioms and is closed under these rules. We call formula $A$ an $L$-theorem, and write $\vdash_{L} A$, when $A \in L$. The smallest logic will be called $\mathrm{B}^{t \forall p}$.

The labels A1-A9 and R1-R5 are as used in chapters 4 and 5 of [11], what we call R6 here is called CR1 there and what we call R7 here is called CR7 there. It will be noted that $\mathrm{R} 6$ and R7 are the rules $t \mathrm{E}$ and $t \mathrm{I}$ stated by Anderson and Belnap when extending their systems with $t$ (for an overview see [3, §R2]). The label RIC stands for "Rule of Intentional Confinement".

From R7, RIC and R6, it is evident that any logic is closed under the rule

\footnotetext{
${ }^{2}$ The rules are read: "from the formulas above the horizontal line (premisses), infer the formula (conclusion) below the horizontal line".
} 


$$
\text { (UG) } \frac{A}{\forall p_{n} A}
$$

of Universal Generalisation. The schemes

- $\forall p_{n} A \wedge \forall p_{n} B \rightarrow \forall p_{n}(A \wedge B)$

- $A \rightarrow \forall p_{n} A$ with $p_{n}$ not free in $A$

are derivable in any logic.

Lemma 2.1. For any formula $A$ with at most one free variable $p_{n}$, and for any closed formulas $B$ and $C$, if $\vdash_{L} B \rightarrow C$ and $\vdash_{L} C \rightarrow B$ then $\vdash_{L} A\left[B / p_{n}\right] \rightarrow A\left[C / p_{n}\right]$ and $\vdash_{L} A\left[C / p_{n}\right] \rightarrow A\left[B / p_{n}\right]$.

Proof. This is by induction on the complexity of $A$. We give only the inductive cases for the quantifiers.

If $A=\forall p_{n} D$, then $\left(\forall p_{n} D\right)\left[B / p_{n}\right]=\forall p_{n} D=\left(\forall p_{n} D\right)\left[C / p_{n}\right]$, so $\vdash_{L}\left(\forall p_{n} D\right)\left[B / p_{n}\right] \rightarrow$ $\left(\forall p_{n} D\right)\left[C / p_{n}\right]$ and $\vdash_{L}\left(\forall p_{n} D\right)\left[C / p_{n}\right] \rightarrow\left(\forall p_{n} D\right)\left[B / p_{n}\right]$ by axiom A1.

If $A=\forall p_{m} D$ with $m \neq n$, then $\left(\forall p_{m} D\right)\left[B / p_{n}\right]=\forall p_{m}\left(D\left[B / p_{n}\right]\right)$ as $B$ is closed, and similarly for $C$. Then we have

$$
\begin{array}{lll}
\text { 1. } & \vdash_{L} \forall p_{m} D\left[B / p_{n}\right] \rightarrow D\left[B / p_{n}\right] & \text { A10 } \\
\text { 2. } & \vdash_{L} D\left[B / p_{n}\right] \rightarrow D\left[C / p_{n}\right] & \text { Induction Hypothesis } \\
\text { 3. } & \vdash_{L} \forall p_{m} D\left[B / p_{n}\right] \rightarrow D\left[C / p_{n}\right] & \text { 1, R3, 2, R1 } \\
\text { 4. } & \vdash_{L} \forall p_{m} D\left[B / p_{n}\right] \rightarrow \forall p_{m} D\left[C / p_{n}\right] & \text { 4, RIC. }
\end{array}
$$

Similarly $\vdash_{L} \forall p_{m} D\left[C / p_{n}\right] \rightarrow \forall p_{m} D\left[B / p_{n}\right]$.

To consider some of the relevant (and irrelevant) logics that have been discussed in the literature, we list some optional axioms below.

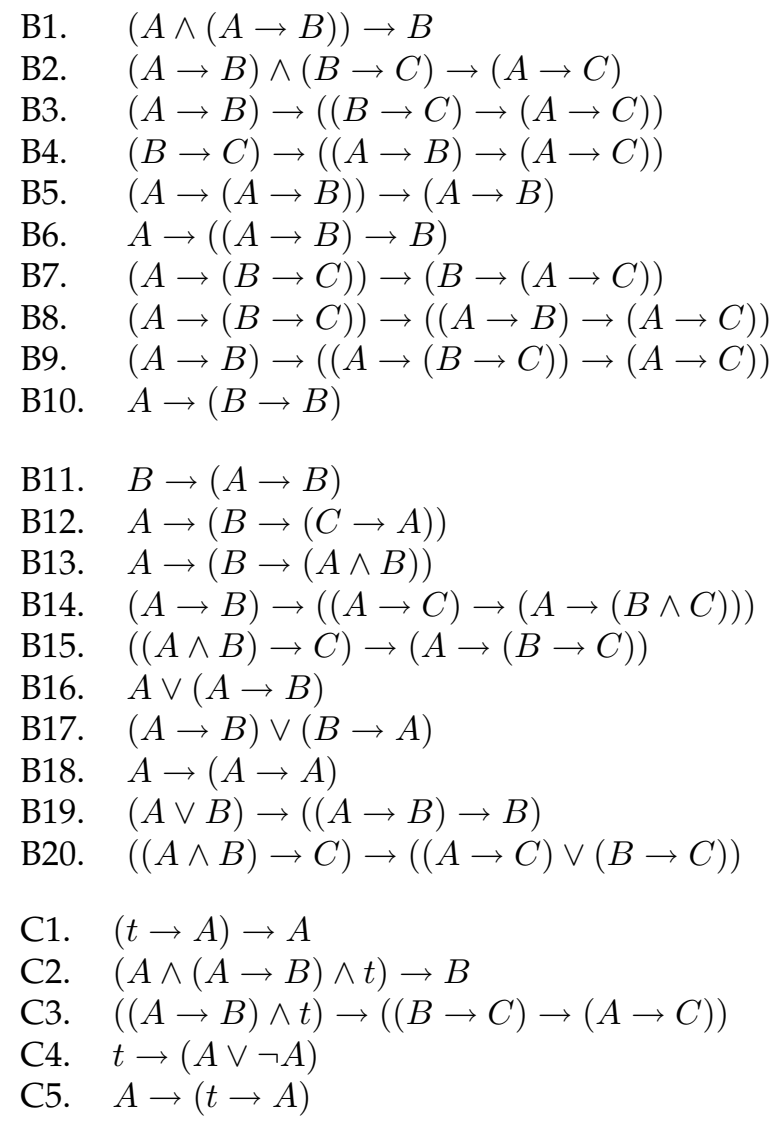




$$
\begin{array}{ll}
\text { D1. } & ((A \wedge B) \rightarrow C) \rightarrow((A \wedge \neg C) \rightarrow \neg B) \\
\text { D2. } & A \vee \neg A \\
\text { D3. } & (A \rightarrow \neg A) \rightarrow \neg A \\
\text { D4. } & (A \rightarrow \neg B) \rightarrow(B \rightarrow \neg A) \\
\text { D5. } & B \rightarrow(A \vee \neg A) \\
\text { D6. } & A \rightarrow(\neg A \rightarrow B) \\
\text { D7. } & \neg(A \rightarrow B) \rightarrow(B \rightarrow A) \\
\text { D8. } & (A \rightarrow \neg(B \rightarrow C)) \rightarrow(\neg B \rightarrow \neg A) \\
\text { E1. } & \forall p_{n}(A \rightarrow B) \rightarrow\left(\forall p_{n} A \rightarrow \forall p_{n} B\right) \\
\text { E2. } & \forall p_{n}(A \vee B) \rightarrow\left(A \vee \forall p_{n} B\right), \text { where } p_{n} \text { is not free in } A . \\
\text { E3. } & (\forall p(p \rightarrow p) \rightarrow A) \rightarrow A
\end{array}
$$

The axioms B1-B20, C1-C5 and D1-D8 are taken directly from Chapter 4 of [11], though with some labeling differences in the case of the $t$ axioms $\mathrm{C} 1-\mathrm{C} 5 . \mathrm{R}^{t \forall p}$ is the smallest logic containing B3-B6, D4, E1 and E2. E3 is derivable in $\mathrm{R}^{t \forall p}$. RP is $\mathrm{R}^{t \forall p}$ without $\mathrm{R} 6$ and $\mathrm{R} 7$, in the language without the constant $t$.

Any logic containing E1 also contains

$$
\forall p_{n}(A \rightarrow B) \rightarrow\left(A \rightarrow \forall p_{n} B\right)
$$

whenever $p_{n}$ is not free in $A$.

Now if $\Sigma$ is any subset of this collection of optional axiom schemas, let $L_{\Sigma}$ be the smallest logic that includes all instances of the members of $\Sigma$. A logic of the form $L_{\Sigma}$ will be called inductively generated. Theoremhood in an inductively generated logic is determined by finite proof sequences: $\vdash_{L_{\Sigma}} A$ iff there is a finite sequence $A_{0}, \ldots, A_{n}=A$ such that each $A_{i}$ is either an instance of A1-A10 or of a member of $\Sigma$, or is derivable from earlier members of the sequence by one of the rules R1-R7, RIC. Using this fact, we can show

Lemma 2.2. Every inductively defined logic is closed under the rules

$$
\begin{aligned}
& \text { RIC(con): } \frac{A \rightarrow B\left[c / p_{n}\right]}{A \rightarrow \forall p_{n} B} \text { if } c \text { is not in } A \text { or } B \text {, and } p_{n} \text { is not free in } A . \\
& \text { UG(con): } \frac{A\left[c / p_{n}\right]}{\forall p_{n} A} \text { if } c \text { is not in } A . \\
& \text { Sub: } \quad \frac{A\left[c_{m_{0}} / p_{m_{0}}, \ldots, c_{m_{n}} / p_{m_{n}}\right]}{A} \text { if } c_{m_{0}}, \ldots, c_{m_{n}} \text { are distinct and not in } A .
\end{aligned}
$$

Proof. The derivations of RIC(con) and UG(con) are similar to Lemmas 6.6 and Corollary 6.7 of [8], using the finite proof-sequence characterisation of theoremhood in the logic.

For Sub, if $\vdash A\left[c_{m_{0}} / p_{m_{0}}, \ldots, c_{m_{n}} / p_{m_{n}}\right]$ then $\vdash \forall p_{m_{n}} A\left[c_{m_{0}} / p_{m_{0}}, \ldots, c_{m_{n-1}} / p_{m_{n-1}}\right]$ by $\mathrm{UG}$ (con), and hence $\vdash A\left[c_{m_{0}} / p_{m_{0}}, \ldots, c_{m_{n-1}} / p_{m_{n-1}}\right]$ from A10. Repeating that argument $n$ times leads to $\vdash A$.

\section{Semantics}

Our models use structures of the form $\langle K, 0, R, *\rangle$, where $K$ is some set (of worlds or setups, or situations ...), 0 is a subset of $K$ (the regular, or base worlds), $R$ is a ternary relation on $K$ and $*$ is a unary function on $K$. We write $a \leq b$ to mean that there is some $x \in 0$ such that $R x a b$. A set $P \subseteq K$ is hereditary if it is closed upward under this relation, i.e. if $a \in P$ and $a \leq b$ then $b \in P$. We call this a basic model structure if it satisfies:

(P1) 0 is hereditary.

(P2) $\leq$ is reflexive and transitive.

(P3) $R b c d$ and $a \leq b$ implies Racd.

(P4) $a^{* *}=a$.

(P5) $a \leq b$ implies $b^{*} \leq a^{*}$. 
Operations $\Rightarrow$ and - on the powerset $\wp K$ of $K$ are defined by

$$
\begin{aligned}
P \Rightarrow Q & =_{d f} \quad\{a \in K: \forall b, c \in K(\text { Rabc and } b \in P \text { implies } c \in Q)\} \\
-P & ={ }_{d f} \quad\left\{a \in K: a^{*} \notin P\right\}
\end{aligned}
$$

Then $P \Rightarrow Q$ is hereditary by (P3), and $-P$ is hereditary if $P$ is, by (P5).

Now fix a set Prop $\subseteq \wp K$. For any $S \subseteq \wp K$, let

$$
\prod S={ }_{d f} \bigcup\{X \in \text { Prop }: X \subseteq \bigcap S\} .
$$

This operation will be used to interpret the universal quantifiers $\forall p_{n}$. In general $\rceil S \subseteq \bigcap S$, and if $\bigcap S \in$ Prop, then $\prod S=\bigcap S$. But it is also possible to have $\Pi S \in$ Prop while $\bigcap S \notin$ Prop. If $S \subseteq$ Prop and $\Pi S \in$ Prop, then $\Pi S$ is the greatest lower bound of $S$ in the partially ordered set $($ Prop,$\subseteq)$.

By a propositional function, relative to Prop, we will mean a function from Prop ${ }^{\omega}$ to Prop. From such functions $\varphi, \psi:$ Prop $^{\omega} \rightarrow \operatorname{Prop}$ we specify new functions $\varphi \cap \psi, \varphi \cup \psi, \varphi \Rightarrow \psi$, $-\varphi$ and $\forall_{n} \varphi$ on Prop ${ }^{\omega}$. For the definition of $\forall_{n} \varphi$ we need functions that "update" a variable assignment $f$, so we write $f[P / n]$ for the function that is identical to $f$ except that it assigns the value $P$ to $n$. Now we put

$$
\begin{array}{rll}
(\varphi \cap \psi) f & =_{d f} & (\varphi f) \cap(\psi f) \\
(\varphi \cup \psi) f & { }_{d f} & (\varphi f) \cup(\psi f) \\
(\varphi \Rightarrow \psi) f & =_{d f} & (\varphi f) \Rightarrow(\psi f) \\
(-\varphi) f & =_{d f} & -(\varphi f) \\
\left(\forall_{n} \varphi\right) f & { }_{d f} & \prod_{P \in \text { Prop }}(\varphi f[P / n]) .
\end{array}
$$

$\mathrm{A} \mathrm{B}^{t \forall p}$-model structure, or just model structure, can now be defined as a structure

$$
\mathscr{K}=\langle K, 0, R, *, \text { Prop, PropFun }\rangle
$$

such that $\langle K, 0, R, *\rangle$ is a basic model stucture, Prop is a set of hereditary subsets of $K$, and PropFun is a set of functions from Prop ${ }^{\omega}$ to Prop, satisfying the following conditions:

CProp: $0 \in$ Prop and if $X$ and $Y$ are in Prop, then $X \cap Y \in$ Prop, $X \Rightarrow Y \in$ Prop and $-X \in$ Prop.

CTee: The function $\varphi_{t}$ is in PropFun, where $\varphi_{t}(f)=0$ for all $f \in$ Prop $^{\omega}$.

CEval: The evaluation function $\varphi_{n}$ is in PropFun for each $n \in \omega$, where $\varphi_{n}(f)=f(n)$ for all $f \in \operatorname{Prop}^{\omega}$.

CImp: If $\varphi, \psi \in$ PropFun, then $\varphi \Rightarrow \psi \in$ PropFun.

CConj: If $\varphi, \psi \in$ PropFun, then $\varphi \cap \psi \in$ PropFun.

CNeg: If $\varphi \in$ PropFun, then $-\varphi \in$ PropFun.

CAll: If $\varphi \in$ PropFun, then $\forall_{n} \varphi \in$ PropFun for all $n \in \omega$.

The condition CProp, which clarifies some of the structure of Prop, is derivable from the others:

Lemma 3.1. If PropFun satisfies the conditions CTee, CEval, CImp, CConj and CNeg, then Prop satisfies CProp.

Proof. By CTee it must be that $0 \in$ Prop. Now take any $P, Q \in$ Prop and consider some $f \in$ Prop $^{\omega}$ such that $f(1)=P$ and $f(2)=Q$. By CEval $\varphi_{1}, \varphi_{2} \in$ PropFun and so by CConj, $\varphi_{1} \cap \varphi_{2} \in$ PropFun. Now $\left(\varphi_{1} \cap \varphi_{2}\right) f=\varphi_{1} f \cap \varphi_{2} f=f(1) \cap f(2)=P \cap Q$, hence $P \cap Q \in$ Prop. Similar arguments using CImp or CNeg show that $P \Rightarrow Q \in$ Prop and $-P \in$ Prop.

$\mathrm{A} \mathrm{B}^{t \forall p}$-model, or just model, is a structure

$$
\mathcal{M}=\langle\mathscr{K}, V\rangle
$$

where $\mathscr{K}$ is a $\mathrm{B}^{t \forall p}$-model sructure, and $V: C o n \rightarrow$ Prop is a function (providing a valuation of the propositional constants) such that: 
CMod: For any propositional constant $c \in$ Con, the constant function $\varphi_{c}$ is in PropFun, where $\varphi_{c}(f)=V(c)$ for all $f \in$ Prop $^{\omega}$.

Each model has a truth/satisfaction relation $\mathcal{M}, a, f \models A$ between worlds $a \in K$, variable assignments $f \in \operatorname{Prop}^{\omega}$, and formulas $A$. This is defined for each $a$ and $f$ by induction on the complexity of $A$, and uses the notion of the truth set of $A$ under $f$ as the set $|A|^{\mathcal{M}} f$ of worlds at which $A$ is true, i.e.

$$
|A|^{\mathcal{M}} f={ }_{d f}\{b \in K: \mathcal{M}, b, f \models A\} .
$$

The inductive definition of $\mid=$ is as follows.

- $\mathcal{M}, a, f \models c$ iff $a \in V(c)$

- $\mathcal{M}, a, f \models p_{n}$ iff $a \in f(n)$

- $\mathcal{M}, a, f \models t$ iff $a \in 0$

- $\mathcal{M}, a, f \models \neg A$ iff $\mathcal{M}, a^{*}, f \not \models A$

- $\mathcal{M}, a, f \models A \wedge B$ iff $\mathcal{M}, a, f \models A$ and $\mathcal{M}, a, f \models B$

- $\mathcal{M}, a, f \models A \rightarrow B$ iff $\forall b \forall c(\mathcal{M}, b, f \models A$ and $R a b c$ implies $\mathcal{M}, c, f \models B)$

- $\mathcal{M}, a, f \models \forall p_{n} A$ iff there is some $X \in P$ Prop such that $a \in X$ and $X \subseteq \bigcap_{P \in P \text { Prop }}|A|^{\mathcal{M}} f[P / n]$.

These truth conditions could alternatively be stated as an inductive definition of the truth sets $|A|^{\mathcal{M}} f$ :

- $|c|^{\mathcal{M}} f=V(c)$

- $\left|p_{n}\right|^{\mathcal{M}} f=f(n)$

- $|t|^{\mathcal{M}} f=0$

- $|\neg A|^{\mathcal{M}} f=-|A|^{\mathcal{M}} f$

- $|A \wedge B|^{\mathcal{M}} f=|A|^{\mathcal{M}} f \cap|B|^{\mathcal{M}} f$

- $|A \rightarrow B|^{\mathcal{M}} f=|A|^{\mathcal{M}} f \Rightarrow|B|^{\mathcal{M}} f$

- $\left|\forall p_{n} A\right|^{\mathcal{M}} f=\prod_{P \in \text { Prop }}|A|^{\mathcal{M}} f[P / n]=\forall_{n}|A|^{\mathcal{M}} f$.

It turns out that that the map $f \mapsto|A|^{\mathcal{M}} f$ that interprets $A$ is a propositional function in the model, i.e. a member of Propfun. To show this, for each formula $A$ we define the propositional function $\varphi_{A}^{\mathcal{M}}$ inductively on the complexity of $A$ :

- $\varphi_{c}^{\mathcal{M}}=\varphi_{c}$

- $\varphi_{p_{n}}^{\mathcal{M}}=\varphi_{n}$

- $\varphi_{t}^{\mathcal{M}}=\varphi_{t}$

- $\varphi_{\neg A}^{\mathcal{M}}=-\varphi_{A}^{\mathcal{M}}$

- $\varphi_{A \wedge B}^{\mathcal{M}}=\varphi_{A}^{\mathcal{M}} \cap \varphi_{B}^{\mathcal{M}}$

- $\varphi_{A \rightarrow B}^{\mathcal{M}}=\varphi_{A}^{\mathcal{M}} \Rightarrow \varphi_{B}^{\mathcal{M}}$

- $\varphi_{\forall p_{n} A}^{\mathcal{M}}=\forall_{n} \varphi_{A}^{\mathcal{M}}$.

Note that each $\varphi_{A}^{\mathcal{M}}$ is indeed in PropFun by the conditions CMod, CEval, CTee, CNeg, CConj, CImp and CAll.

Lemma 3.2. Let $A$ be an arbitrary formula. Then in any model, $\varphi_{A}^{\mathcal{M}} f=|A|^{\mathcal{M}} f$ for any $f \in$ Prop ${ }^{\omega}$. Hence $|A|^{\mathcal{M}} f$ is a proposition, i.e. a member of Prop.

Proof. By induction on the complexity of $A$. The base cases are

$$
\begin{gathered}
\varphi_{c}^{\mathcal{M}} f=\varphi_{c} f=V(c)=|c|^{\mathcal{M}} f \\
\varphi_{p_{n}}^{\mathcal{M}} f=\varphi_{n} f=f(n)=\left|p_{n}\right|^{\mathcal{M}} f \\
\varphi_{t}^{\mathcal{M}} f=\varphi_{t} f=0=|t|^{\mathcal{M}} f
\end{gathered}
$$

The inductive cases follow similarly from the correspondence of the definitions. 
Since Prop consists of hereditary sets, we get

Corollary 3.3 (Hereditariness). In any model, for any formula $A$, if $a \leq b$ and $\mathcal{M}, a, f \models A$ then $\mathcal{M}, b, f \models A$.

We say that a formula $A$ is satisfied by the assignment $f$ in the model $\mathcal{M}$ when $\mathcal{M}, a, f=A$ for all base worlds $a \in 0 . A$ is valid in the model $\mathcal{M}$, written $\mathcal{M}=A$, if it is satisfied by every assignment $f \in \operatorname{Prop}^{\omega}$. $A$ is valid on the model-structure $\mathscr{K}$, written $\mathscr{K} \models A$, if it is valid in every model based on $\mathscr{K}$.

The following is proved as in [10, Lemmas 2 and 3].

Lemma 3.4 (Semantic Entailment). For any model $\mathcal{M}$, the formula $A \rightarrow B$ is satisfied by $f$ in $\mathcal{M}$ iff for any world $a \in K, \mathcal{M}, a, f=A$ implies $\mathcal{M}, a, f \models B$ i.e. iff $|A|^{\mathcal{M}} f \subseteq|B|^{\mathcal{M}} f$.

Next we show that the satisfaction relation depends only on the value assignment to free variables.

Lemma 3.5. For any formula $A$ and $f, g \in$ Prop $^{\omega}$, if $f$ and $g$ agree on the free variables of $A$ then $|A|^{\mathcal{M}} f=|A|^{\mathcal{M}} g$ (and hence $\varphi_{A}^{\mathcal{M}} f=\varphi_{A}^{\mathcal{M}} g$ by Lemma 3.2).

Proof. By induction on the complexity of $A$. If $A=p_{n}$, then as $f$ and $g$ agree on the free variable $p_{n},\left|p_{n}\right|^{\mathcal{M}} f=f(n)=g(n)=\left|p_{n}\right|^{\mathcal{M}} g$.

The cases of $A=c \in C$ on and $A=t$, are straightforward, as are the induction cases for $\wedge, \rightarrow$, and $\neg$.

For $A=\forall p_{n} B$ :

$$
\left|\forall p_{n} B\right|^{\mathcal{M}} f=\prod_{P \in \text { Prop }}|B|^{\mathcal{M}} f[P / n]=\prod_{P \in \text { Prop }}|B|^{\mathcal{M}} g[P / n]=\left|\forall p_{n} B\right|^{\mathcal{M}} g
$$

by induction hypothesis as, for each $P, f[P / n]$ and $g[P / n]$ must clearly agree on all free variables in $B$ by assumption.

The semantics of formula-substitution is characterised by updating of variable assignments, in a similar manner to first-order predicate logic (see for example [8, Lemma 7.1]):

Lemma 3.6. In any model, for any $f \in$ Prop $^{\omega}$, formulas $A, B$, and variable $p_{n}$, if $p_{n}$ is free for $B$ in $A$, then $\left|A\left[B / p_{n}\right]\right|^{\mathcal{M}} f=|A|^{\mathcal{M}} f\left[|B|^{\mathcal{M}} f / n\right]$.

Proof. First note that $|A|^{\mathcal{M}} f\left[|B|^{\mathcal{M}} f / n\right]$ is indeed a well-defined notion, as $|B|^{\mathcal{M}} f \in$ Prop by Lemma 3.2, hence $f\left[|B|^{\mathcal{M}} f / n\right] \in \operatorname{Prop}^{\omega}$. We will let $f^{\prime}=f\left[|B|^{\mathcal{M}} f / n\right]$ and proceed by induction on the complexity of $A$.

For $A=p_{n}$,

$$
\left|p_{n}\left[B / p_{n}\right]\right|^{\mathcal{M}} f=|B|^{\mathcal{M}} f=f^{\prime}(n)=\left|p_{n}\right|^{\mathcal{M}} f^{\prime}
$$

while for $A=p_{m}$ with $m \neq n$,

$$
\left|p_{m}\left[B / p_{n}\right]\right|^{\mathcal{M}} f=\left|p_{m}\right|^{\mathcal{M}} f=f(m)=f^{\prime}(m)=\left|p_{m}\right|^{\mathcal{M}} f^{\prime} .
$$

The cases of $A=c \in C$ on and $A=t$, and the induction cases for $\wedge, \rightarrow$, and $\neg$, are left to the reader.

For $A=\forall p_{m} C$ where $p_{n}$ does not occur free in $A$,

$$
\left|\left(\forall p_{m} C\right)\left[B / p_{n}\right]\right|^{\mathcal{M}} f=\left|\forall p_{m} C\right|^{\mathcal{M}} f=\left|\forall p_{m} C\right|^{\mathcal{M}} f^{\prime}
$$

follows by Lemma 3.5 as $f$ and $f^{\prime}$ differ only in their assignment to $p_{n}$, hence they agree on the free variables of $A=\forall p_{m} C$. 
For $A=\forall p_{m} C$ with $p_{n}$ free in $A$,

$$
\begin{aligned}
\left|\left(\forall p_{m} C\right)\left[B / p_{n}\right]\right|^{\mathcal{M}} f & =\left|\forall p_{m}\left(C\left[B / p_{n}\right]\right)\right|^{\mathcal{M}} f \\
& =\prod_{Q \in \text { Prop }}\left|C\left[B / p_{n}\right]\right|^{\mathcal{M}} f[Q / m] \\
& =\prod_{Q \in \text { Prop }}|C|^{\mathcal{M}} f[Q / m]\left[|B|^{\mathcal{M}} f[Q / m] / n\right] \\
& =\prod_{Q \in \operatorname{Prop}}|C|^{\mathcal{M}} f[Q / m]\left[|B|^{\mathcal{M}} f / n\right] \\
& =\prod_{Q \in \operatorname{Prop}}|C|^{\mathcal{M}} f\left[|B|^{\mathcal{M}} f / n\right][Q / m] \\
& =|\forall|^{\mathcal{M}} f^{\prime}[Q / m]
\end{aligned}
$$

with each step justified as follows:

(a) as $p_{n}$ is assumed free for $B$ in $A=\forall p_{m} C$.

(b) by the truth condition for $\forall p_{m}$.

(c) by induction hypothesis.

(d) as for any $Q,|B|^{\mathcal{M}} f=|B|^{\mathcal{M}} f[Q / m]$. This holds because the assumption that $p_{n}$ is free for $B$ in $A$, implies that $B$ has no free variables that would become bound in $A\left[B / p_{n}\right]=\left(\forall p_{m} C\right)\left[B / p_{n}\right]$. So in particular $p_{m}$ does not occur free in $B$. Thus $f$ and $f[Q / m]$ agree on the free variables of $B$ (for any $Q$ ), hence by Lemma $3.5|B|^{\mathcal{M}} f=$ $|B|^{\mathcal{M}} f[Q / m]$.

(e) as $m \neq n$ (else $p_{n}$ would not occur free in $A$ ).

(f) by the definition of $f^{\prime}$.

(g) by the truth condition for $\forall p_{m}$.

Theorem 3.7 ( $\mathrm{B}^{t \forall p}$-Soundness). For any formula $A$, if $A$ is a $B^{t \forall p}$-theorem, then $A$ is valid in all $B^{t \forall p}$-model structures.

Proof. Let $\mathcal{M}$ be any model on a $\mathrm{B}^{t \forall p}$-model structure. We need to show that the axioms A1-A10 are valid in $\mathcal{M}$, and that the rules R1-R7, RIC preserve this validity. For A1-A9 and R1-R7, this proceeds as in $[11, \S 4.5]$.

For A10, suppose $\mathcal{M}, a, f \models \forall p_{n} A$. Let $B$ be a formula such that no free variable in $B$ becomes bound in $A\left[B / p_{n}\right]$ (i.e. $p_{n}$ is free for $B$ in $A$ ) and define $f^{\prime}=f\left[|B|^{\mathcal{M}} f / n\right]$. Now by the truth condition for $\forall p_{n}$ there is some $X \in \operatorname{Prop}$ such that $X \subseteq \bigcap_{P \in \text { Prop }}|A|^{\mathcal{M}} f[P / n]$ and $a \in X$. In particular, if we take $P$ as $|B|^{\mathcal{M}} f$, then we see $X \subseteq|A|^{\mathcal{M}} f^{\prime}$. So $a \in\left|A\left[B / p_{n}\right]\right|^{\mathcal{M}} f$ by Lemma 3.6, i.e. $\mathcal{M}, a, f \models A\left[B / p_{n}\right]$. Hence by Semantic Entailment (Lemma 3.4) and the arbitrary choice of $f, \forall p_{n} A \rightarrow A\left[B / p_{n}\right]$ is valid in $\mathcal{M}$.

For RIC, Suppose $A \rightarrow B$ is valid in $\mathcal{M}$, where $p_{n}$ does not occur free in $A$. By the definition of validity and Semantic Entailment we have that $|A|^{\mathcal{M}} g \subseteq|B|^{\mathcal{M}} g$ for any $g \in$ Prop $^{\omega}$, so

$$
\bigcap_{P \in \text { Prop }}|A|^{\mathcal{M}} f[P / n] \subseteq \bigcap_{P \in \text { Prop }}|B|^{\mathcal{M}} f[P / n] .
$$

Now as $p_{n}$ is not free in $A$, Lemma 3.5 ensures that $|A|^{\mathcal{M}} f=|A|^{\mathcal{M}} f[P / n]$ for any $P \in$ Prop. It follows that

$$
|A|^{\mathcal{M}} f \subseteq \bigcap_{P \in \text { Prop }}|B|^{\mathcal{M}} f[P / n] .
$$

Hence as $|A|^{\mathcal{M}} f \in$ Prop by Lemma 3.2,

$$
|A|^{\mathcal{M}} f \subseteq \bigcup\left\{Q \in \operatorname{Prop}: Q \subseteq \bigcap_{P \in \text { Prop }}|B|^{\mathcal{M}} f[P / n]\right\},
$$

i.e. $|A|^{\mathcal{M}} f \subseteq\left|\forall p_{n} B\right|^{\mathcal{M}} f$. So by Semantic Entailment, $A \rightarrow \forall p_{n} B$ is valid in $\mathcal{M}$. 


\section{Completeness of $\mathbf{B}^{t \forall p}$}

Fix an arbitrary logic $L$. We construct a characteristic model $\mathcal{M}_{L}$ that validates precisely the theorems of $L$. This adapts the Henkin-style constructions of [10] and [11, §4.6], in which the points of the model are certain theories, i.e. sets of formulas with suitable proof-theoretic closure conditions. We take much of the propositional-logic aspect of the construction as known from these references, and focus on its extension to our interpretation of the quantifiers.

For sets of formulae $\Gamma, \Delta$, we write $\Gamma \Rightarrow_{L} \Delta$ if there are some $A_{1}, \ldots, A_{n} \in \Gamma$ and $B_{1}, \ldots, B_{m} \in \Delta$ such that $\vdash_{L} A_{1} \wedge \ldots \wedge A_{n} \rightarrow B_{1} \vee \ldots \vee B_{m}$. The pair $(\Gamma, \Delta)$ is L-independent when $\Gamma \nexists_{L} \Delta$. $\Gamma$ is an L-theory if for any formula $B, \Gamma \Rightarrow_{L}\{B\}$ implies $B \in \Gamma$. An L-theory $\Gamma$ is prime when $A \vee B \in \Gamma$ implies $A \in \Gamma$ or $B \in \Gamma$, and regular when $\vdash_{L} A$ implies $A \in \Gamma$. The appropriate version of Lindenbaum's Lemma in this context is

Lemma 4.1. [11, Lemma 4.3] If $(\Gamma, \Delta)$ is L-independent, there there exists some prime L-theory $\Gamma^{\prime}$ such that $\Gamma \subseteq \Gamma^{\prime}$ and $\left(\Gamma^{\prime}, \Delta\right)$ is L-independent.

Corollary 4.2. If $\nvdash_{L} A$, there is a regular prime $L$-theory not containing $A$.

Proof. Take $\Gamma=\left\{B: \vdash_{L} B\right\}$ and $\Delta=\{A\}$ in the Lemma.

Now let $K_{L}$ be the set of all prime L-theories and $0_{L}$ be the set of all regular prime L-theories. Then for each closed formula $A$, define $\|A\|_{L}=_{d f}\left\{a \in K_{L}: A \in a\right\}$. Put

$$
\operatorname{Prop}_{L}=\left\{\|A\|_{L}: A \text { is a closed formula }\right\} .
$$

From this definition we get an analogue of the Semantic Entailment Lemma 3.4:

Lemma 4.3. For any closed formulas $A, B$ we have that $\|A\|_{L} \subseteq\|B\|_{L}$ iff $\vdash_{L} A \rightarrow B$.

Proof. Suppose $\|A\|_{L} \subseteq\|B\|_{L}$. If $\forall_{L} A \rightarrow B$ then $(\{A\},\{B\})$ is an L-independent pair. So by Lemma 4.1 there is some prime L-theory $\Gamma$ exdending $\{A\}$ such that $(\Gamma,\{B\})$ is an Lindependent pair. Therefore $\Gamma \in K_{L}$ (as $\Gamma$ is a prime L-theory), $A \in \Gamma$ and $B \notin \Gamma$. But then $\Gamma \in\|A\|_{L}$ and $\Gamma \notin\|B\|_{L}$ contradicting $\|A\|_{L} \subseteq\|B\|_{L}$. Hence $\vdash_{L} A \rightarrow B$.

Conversely, suppose $\vdash_{L} A \rightarrow B$ and consider any $a \in\|A\|_{L}$. Then $A \in a$, and as $a$ is an L-theory (closed under L-implication), $B \in a$, so $a \in\|B\|_{L}$. Hence $\|A\|_{L} \subseteq\|B\|_{L}$.

We need a particular way of naming members of $\operatorname{Prop}_{L}$, since for a given closed $B$ there will be infinitely many closed $B^{\prime}$ with $\|B\|_{L}=\left\|B^{\prime}\right\|_{L}$. So we assume there is some fixed enumeration of all the closed formulas of our language, and for each $Q \in \operatorname{Prop}_{L}$, define $B_{Q}$ to be the first formula in this enumeration such that $\left\|B_{Q}\right\|_{L}=Q$. Then for any formula $A$ and $Q_{0}, Q_{1}, \ldots \in \operatorname{Prop}_{L}$, let

$$
A\left[Q_{0} / p_{0}, \ldots, Q_{n} / p_{n}, \ldots\right]={ }_{d f} A\left[B_{Q_{0}} / p_{0}, \ldots, B_{Q_{n}} / p_{n}, \ldots\right] .
$$

This definition may be restricted to single substitutions $A\left[Q / p_{n}\right]$ or to any finite substitution $A\left[Q_{0} / p_{n_{0}}, \ldots, Q_{m} / p_{n_{m}}\right]$ in the obvious way. We will also allow ourselves the liberty of specifying mixed proposition and formula substitutions, e.g. where $Q \in \operatorname{Prop}$ and $C$ is a formula, $A\left[Q / p_{m}, C / p_{n}\right]=A\left[B_{Q} / p_{m}, C / p_{n}\right]$.

To show that our choice of an enumeration of closed formulas does not really matter, we have

Lemma 4.4. For any formula $A$ with at most one free variable $p_{n}$, and for any closed formulas $B$ and $C$, if $\|B\|_{L}=\|C\|_{L}$ then $\left\|A\left[B / p_{n}\right]\right\|_{L}=\left\|A\left[C / p_{n}\right]\right\|_{L}$.

Proof. If $\|B\|_{L}=\|C\|_{L}$ then by Lemma $4.3 \vdash_{L} B \rightarrow C$ and $\vdash_{L} C \rightarrow B$. Now by Lemma 2.1 we get $\vdash_{L} A\left[B / p_{n}\right] \rightarrow A\left[C / p_{n}\right]$ and $\vdash_{L} A\left[C / p_{n}\right] \rightarrow A\left[B / p_{n}\right]$. Then $\left\|A\left[B / p_{n}\right]\right\|_{L}=$ $\left\|A\left[C / p_{n}\right]\right\|_{L}$ by Lemma 4.3 again.

Corollary 4.5. For any formula $A$, given closed formulas $B_{0}, \ldots, B_{n}, \ldots$ and $C_{0}, \ldots, C_{n}, \ldots$, if $\left\|B_{i}\right\|_{L}=\left\|C_{i}\right\|_{L}$ for all $i \in \omega$ then $\left\|A\left[B_{0} / p_{0}, \ldots, B_{n} / p_{n}, \ldots\right]\right\|_{L}=\left\|A\left[C_{0} / p_{0}, \ldots, C_{n} / p_{n}, \ldots\right]\right\|_{L}$. 
Proof. Take some $m \in \omega$ such that $A\left[B_{0} / p_{0}, \ldots, B_{m} / p_{m}\right]=A\left[B_{0} / p_{0}, \ldots, B_{n} / p_{n}, \ldots\right]$ (i.e. all the free variables of $A$ occur among $\left.p_{0}, \ldots, p_{m}\right)$. Also, given each $B_{i}$ or $C_{i}$ is a closed formula, it does not matter what order we substitute them into $A$. In particular, for any $i$,

$$
A\left[B_{0} / p_{0}, \ldots\right]=A\left[B_{0} / p_{0}, \ldots, B_{i-1} / p_{i-1}, B_{i+1} / p_{i+1}, \ldots\right]\left[B_{i} / p_{i}\right] .
$$

Now using this information and Lemma 4.4 we see that

$$
\begin{aligned}
& \left\|A\left[B_{0} / p_{0}, \ldots, B_{n} / p_{n}, \ldots\right]\right\|_{L} \\
& =\left\|A\left[B_{0} / p_{0}, \ldots, B_{m} / p_{m}\right]\right\|_{L} \\
& =\left\|A\left[B_{1} / p_{1}, \ldots, B_{m} / p_{m}\right]\left[B_{0} / p_{0}\right]\right\|_{L} \\
& =\left\|A\left[B_{1} / p_{1}, \ldots, B_{m} / p_{m}\right]\left[C_{0} / p_{0}\right]\right\|_{L} \\
& =\left\|A\left[C_{0} / p_{0}, B_{1} / p_{1}, \ldots, B_{m} / p_{m}\right]\right\|_{L} \\
& \vdots \\
& =\left\|A\left[C_{0} / p_{0}, C_{1} / p_{1}, \ldots, B_{m} / p_{m}\right]\right\|_{L} \\
& \vdots \\
& =\left\|A\left[C_{0} / p_{0}, \ldots, C_{m} / p_{m}\right]\right\|_{L} \\
& =\left\|A\left[C_{0} / p_{0}, \ldots, C_{n} / p_{n}, \ldots\right]\right\|_{L} .
\end{aligned}
$$

Given $f \in \operatorname{Prop}_{L}^{\omega}$, for any formula $A$, let

$$
A^{f}={ }_{d f} A\left[f(0) / p_{0}, \ldots, f(n) / p_{n}, \ldots\right]=A\left[B_{f(0)} / p_{0}, \ldots, B_{f(n)} / p_{n}, \ldots\right] .
$$

It is clear that if $A$ is closed then $A^{f}$ is just $A$. Furthermore, $A^{f}$ will always be closed, as a free $p_{i}$ in $A$ is replaced by some closed formula $B_{f(i)}$ (where $\left\|B_{f(i)}\right\|_{L}=f(i)$ ). So for any $A$ and $f$ we have $\left\|A^{f}\right\|_{L} \in \operatorname{Prop}_{L}$. The substitution operator $f \mapsto A^{f}$ commutes with the connectives: $(A \wedge B)^{f}=\left(A^{f} \wedge B^{f}\right),(A \rightarrow B)^{f}=\left(A^{f} \rightarrow B^{f}\right)$ and $(\neg A)^{f}=\neg\left(A^{f}\right)$.

Lemma 4.6. If $f(i)=\left\|C_{i}\right\|_{L}$ for all $i \in \omega$, then $\left\|A^{f}\right\|_{L}=\left\|A\left[C_{0} / p_{0}, \ldots, C_{n} / p_{n}, \ldots\right]\right\|_{L}$.

Proof. Take $B_{i}=B_{f(i)}$ in Corollary 4.5.

Now for each formula $A$, define $\varphi_{L}^{A}: \operatorname{Prop}_{L}^{\omega} \rightarrow \operatorname{Prop}_{L}$ by $\varphi_{L}^{A} f=\left\|A^{f}\right\|_{L}$. Put

$$
\operatorname{PropFun}_{L}=\left\{\varphi_{L}^{A}: A \text { is a formula }\right\} .
$$

The canonical L-model structure is

$$
\mathcal{K}_{L}=\left\langle K_{L}, 0_{L}, R_{L}, *_{L}, \text { Prop }_{L}, \operatorname{PropFun}_{L}\right\rangle
$$

where

- $R_{L} a b c$ iff $A \rightarrow B \in a$ and $A \in b$ implies $B \in c$,

- $a^{*_{L}}=\{A: \neg A \notin a\}$ for any $a \in K_{L}$,

and the other items are already defined. The canonical $L$-model is $\mathcal{M}_{L}=\left\langle\mathcal{K}_{L}, V_{L}\right\rangle$, where $V_{L}(c)=\|c\|_{L}$ for all $c \in$ Con.

Now $\mathcal{K}_{L}$ can be shown to be a $\mathrm{B}^{t \forall p}$-model structure. The authors of $[11, \S 4.6]$ show that $\left\langle K_{L}, 0_{L}, R_{L}, *_{L}\right\rangle$ is a basic model structure, in which $a \leq b$ iff $a \subseteq b$. They also show that $0_{L}=\|t\|_{L},\|A\|_{L} \cap\|B\|_{L}=\|A \wedge B\|_{L},\|A\|_{L} \Rightarrow\|B\|_{L}=\|A \rightarrow B\|_{L}$ and $-\|A\|_{L}=\|\neg A\|_{L}$. This implies that $\mathcal{K}_{L}$ satisfies the condition CProp.

For CTee, observe that $\varphi_{L}^{t} \in \operatorname{PropFun}_{L}$, where $\varphi_{L}^{t}: f \mapsto\left\|t^{f}\right\|_{L}$. But as $t$ is a closed formula, $\left\|t^{f}\right\|_{L}=\|t\|_{L}=0_{L}$. So $\varphi_{L}^{t}$ satisfies the definition for $\varphi_{t}$, therefore $\varphi_{t} \in \operatorname{PropFun}_{L}$.

For CEval, we have $\varphi_{L}^{p_{n}} \in \operatorname{PropFun}_{L}$, where $\varphi_{L}^{p_{n}}: f \mapsto\left\|p_{n}{ }^{f}\right\|_{L}$. Now

$$
p_{n}^{f}=p_{n}\left[B_{f(0)} / p_{0}, \ldots, B_{f(n)} / p_{n}, \ldots\right]=B_{f(n)},
$$

so $\varphi_{L}^{p_{n}}: f \mapsto\left\|p_{n}{ }^{f}\right\|_{L}=\left\|B_{f(n)}\right\|_{L}=f(n)$. So $\varphi_{L}^{p_{n}}$ satisfies the definition for $\varphi_{n}$ and therefore $\varphi_{n} \in$ PropFun $_{L}$. 


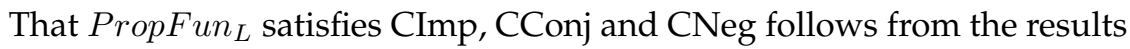

$$
\begin{aligned}
\left(\varphi_{L}^{A} \Rightarrow \varphi_{L}^{B}\right) & =\varphi_{L}^{A \rightarrow B} \\
\left(\varphi_{L}^{A} \cap \varphi_{L}^{B}\right) & =\varphi_{L}^{A \wedge B} \\
-\left(\varphi_{L}^{A}\right) & =\varphi_{L}^{A} .
\end{aligned}
$$

For the first of these,

$$
\left(\varphi_{L}^{A} \Rightarrow \varphi_{L}^{B}\right) f=\left\|A^{f}\right\|_{L} \Rightarrow\left\|B^{f}\right\|_{L}=\left\|A^{f} \rightarrow B^{f}\right\|_{L}=\left\|(A \rightarrow B)^{f}\right\|_{L}=\left(\varphi_{L}^{A \rightarrow B}\right) f,
$$

and the others are similar.

Our main burden is to show that that CAll holds on the canonical model. The following two lemmas are analogous to Lemmas 9.3 and 9.4 of [8].

Lemma 4.7. For any closed formula $\forall p_{n} A$, and any prime L-theory a, we have that $a \in\left\|\forall p_{n} A\right\|_{L}$ iff there is some $X \in \operatorname{Prop}_{L}$ such that $a \in X$ and $X \subseteq\left\|A\left[Q / p_{n}\right]\right\|_{L}$ for all $Q \in \operatorname{Prop}_{L}$, i.e.

$$
\left\|\forall p_{n} A\right\|_{L}=\prod_{Q \in \text { Prop }_{L}}\left\|A\left[Q / p_{n}\right]\right\|_{L} .
$$

Proof. Suppose $a \in\left\|\forall p_{n} A\right\|_{L}$ i.e. $\forall p_{n} A \in a$. Let $X=\left\|\forall p_{n} A\right\|_{L}$. Then $a \in X \in \operatorname{Prop}_{L}$ as $\forall p_{n} A$ is closed. Consider any $Q \in \operatorname{Prop}_{L}$, and recall that $Q=\left\|B_{Q}\right\|_{L}$. Now as $\forall p_{n} A \in a$ and $\vdash_{L} \forall p_{n} A \rightarrow A\left[B_{Q} / p_{n}\right]$ (axiom A10, applicable as $B_{Q}$ is a closed formula), by closure of L-theories under L-implication we get $A\left[B_{Q} / p_{n}\right] \in a$ and hence $a \in\left\|A\left[B_{Q} / p_{n}\right]\right\|_{L}$. So $X \subseteq\left\|A\left[B_{Q} / p_{n}\right]\right\|_{L}=_{d f}\left\|A\left[Q / p_{n}\right]\right\|_{L}$.

Conversely, suppose there is some $X \in \operatorname{Prop}_{L}$ such that $a \in X$ and $X \subseteq\left\|A\left[Q / p_{n}\right]\right\|_{L}$ for all $Q \in \operatorname{Prop}_{L}$. By definition of $\operatorname{Prop}_{L}$, there is some closed formula $B_{X}$ such that $\left\|B_{X}\right\|=X$. Hence $B_{X} \in a$. Choose a constant $c \in C$ on that does not occur in $A$ or $B_{X}$. Let $Q=\|c\|_{L} \in \operatorname{Prop}_{L}$.

Now if $\forall_{L} B_{X} \rightarrow A\left[c / p_{n}\right]$, then $\left(\left\{B_{X}\right\},\left\{A\left[c / p_{n}\right]\right\}\right)$ is an L-independent pair. So by Lemma 4.1 there is some prime L-theory $\Gamma$, extending $\left\{B_{X}\right\}$, such that $\left(\Gamma,\left\{A\left[c / p_{n}\right]\right\}\right)$ is an L-independent pair. So $B_{X} \in \Gamma$, giving $\Gamma \in\left\|B_{X}\right\|_{L}=X$, and $A\left[c / p_{n}\right] \notin \Gamma$, giving $\Gamma \notin\left\|A\left[c / p_{n}\right]\right\|_{L}$. But as $\|c\|_{L}=\left\|B_{Q}\right\|_{L}$ we have that $\left\|A\left[c / p_{n}\right]\right\|_{L}=\left\|A\left[B_{Q} / p_{n}\right]\right\|_{L}$ by Lemma 4.4. Hence $\Gamma \notin\left\|A\left[B_{Q} / p_{n}\right]\right\|_{L}$. But $\left\|A\left[Q / p_{n}\right]\right\|_{L}=_{d f}\left\|A\left[B_{Q} / p_{n}\right]\right\|_{L}=\left\|A\left[c / p_{n}\right]\right\|_{L}$, and as such it must be that $\Gamma \notin\left\|A\left[Q / p_{n}\right]\right\|_{L}$. But $\Gamma \in X$, so $\Gamma$ witnesses $X \not \Perp\|A[Q / p]\|_{L}$, contradicting our original supposition.

Therefore it must be that $\vdash_{L} B_{X} \rightarrow A\left[c / p_{n}\right]$, so by the rule RIC(con) of Lemma 2.2, $\vdash_{L} B_{X} \rightarrow \forall p_{n} A$. So finally, as $a$ is a L-theory and $B_{X} \in a$, closure of L-theories under L-implication gives $\forall p_{n} A \in a$, i.e. $a \in\left\|\forall p_{n} A\right\|_{L}$.

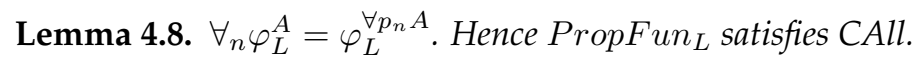

Proof. We begin with a definition. If $f \in \operatorname{Prop}_{L}^{\omega}$ and $n \in \omega$, then

$$
A^{f \backslash n}={ }_{d f} A\left[f(0) / p_{0}, \ldots, f(n-1) / p_{n-1}, p_{n} / p_{n}, f(n+1) / p_{n+1}, \ldots\right]
$$

is the (possibly open) formula which applies the substitution $f$ to all variables except $p_{n}$, which remains unchanged. This satisfies:

$$
\begin{aligned}
& A^{f \backslash n}\left[Q / p_{n}\right]=A^{f[Q / n]} \\
& \forall p_{n}\left(A^{f \backslash n}\right)=\left(\forall p_{n} A\right)^{f} .
\end{aligned}
$$

Equation (4.2) holds because, as was mentioned in the proof of Corollary 4.5, when substituting closed formulas it does not matter in what order they are substituted for their respective variables (as long as we only try substituting once for each variable, which we do in $A^{f \backslash n}\left[Q / p_{n}\right]$ and $A^{f[Q / n]}$ ). 
For equation (4.3) we note that $\forall p_{n}\left(A^{f \backslash n}\right)=\left(\forall p_{n} A\right)^{f \backslash n}$ (for, as $f \backslash n$ leaves $p_{n}$ unchanged, it makes no difference if it is forced to leave it unchanged, as in $\left.\left(\forall p_{n} A\right)^{f \backslash n}\right)$. And clearly $\left(\forall p_{n} A\right)^{f \backslash n}=\left(\forall p_{n} A\right)^{f}$ as $p_{n}$ is not free in $\forall p_{n} A$.

Now for any $\varphi_{L}^{A} \in \operatorname{PropFun}_{L}, f \in \operatorname{Prop}_{L}^{\omega}$ and $n \in \omega$ we have

$$
\begin{aligned}
\left(\forall_{n} \varphi_{L}^{A}\right) f & =\prod_{Q \in \operatorname{Prop}} \varphi_{L}^{A}(f[Q / n]) & & \text { by definition of } \forall_{n} \\
& =\prod_{Q \in \operatorname{Prop}}\left\|A^{f[Q / n]}\right\|_{L} & & \text { by definition of } \varphi_{L}^{A} \\
& =\prod_{Q \in \operatorname{Prop}}\left\|A^{f \backslash n}\left[Q / p_{n}\right]\right\|_{L} & & \text { by (4.2) } \\
& =\left\|\forall p_{n}\left(A^{f \backslash n}\right)\right\|_{L} & & \text { by Lemma } 4.7 \\
& =\left\|\left(\forall p_{n} A\right)^{f}\right\|_{L} & & \text { by (4.3) } \\
& =\left(\varphi_{L}^{\forall p_{n} A}\right) f & & \text { by definition of } \varphi_{L}^{\forall p_{n} A} .
\end{aligned}
$$

Therefore $\forall_{n} \varphi_{L}^{A}=\varphi_{L}^{\forall p_{n} A} \in \operatorname{PropFun}_{L}$, and so CAll holds.

That completes the proof that $\mathcal{K}_{L}$ is a $\mathrm{B}^{t \forall p}$-model structure. To show that $\mathcal{M}_{L}$ is a $\mathrm{B}^{t \forall p}$ model, it remains only to show that it satisfies CMod. But if $c \in C o n$, then by definition, $\varphi_{L}^{c} \in$ PropFun $_{L}$, where, as $c$ is closed,

$$
\varphi_{L}^{c} f={ }_{d f}\left\|c^{f}\right\|_{L}=\|c\|_{L}=_{d f} V_{L}(c) .
$$

Hence $\varphi_{L}^{c}$ satisfies the definition for $\varphi_{c}$, and therefore $\varphi_{c} \in \operatorname{PropFun}_{L}$ as required for CMod.

Lemma 4.9 (Truth Lemma). For any formula $A, \varphi_{A}^{\mathcal{M}_{L}}=\varphi_{L}^{A}$. Equivalently (by Lemma 3.2), for all $f \in \operatorname{Prop}_{L}^{\omega}$ we have $|A|^{\mathcal{M}_{L}} f=\left\|A^{f}\right\|_{L}$, and so for all $a \in K_{L}$ we have that

$$
\mathcal{M}_{L}, a, f \models A \text { iff } A^{f} \in a \text {. }
$$

Proof. By induction on the complexity of $A$. For the proof we write $\mathcal{M}_{L}$ just as $\mathcal{M}$.

For the base cases, by definition we have $\varphi_{p_{n}}^{\mathcal{M}}=\varphi_{n}, \varphi_{c}^{\mathcal{M}}=\varphi_{c}$ and $\varphi_{t}^{\mathcal{M}}=\varphi_{t}$. But we saw above that $\varphi_{n}=\varphi_{L}^{p_{n}}, \varphi_{c}=\varphi_{L}^{c}$ and $\varphi_{t}=\varphi_{L}^{t}$, so the Lemma holds when $A=p_{n}, c$ or $t$.

For the inductive case of $A=B \rightarrow C$, we have

$$
|B \rightarrow C|^{\mathcal{M}}=|B|^{\mathcal{M}} \Rightarrow|C|^{\mathcal{M}}=\varphi_{L}^{B} \Rightarrow \varphi_{L}^{C}
$$

by induction hypothesis, which equals $\varphi_{L}^{B \rightarrow C}$ by the first equation of (4.1). The cases of $A=B \wedge C$ and $A=\neg B$ are similar.

Finally, for the case $A=\forall p_{n} B:\left|\forall p_{n} B\right|^{\mathcal{M}}=\forall_{n}|B|^{\mathcal{M}}=\forall_{n} \varphi_{L}^{B}$ by induction hypothesis, which equals $\varphi_{L}^{\forall p_{n} B}$ as in the proof of Lemma 4.8 above.

Corollary 4.10. $\vdash_{L}$ A implies $\mathcal{M}_{L} \models A$.

Proof. Let $\vdash_{L} A$, and choose $n$ such that the free variables of $A$ are among $p_{0}, \ldots, p_{n}$. By the rule UG, $\vdash_{L} \forall p_{0} \cdots \forall p_{n} A$. Hence using axiom A10, for any closed formulas $B_{0}, \ldots, B_{n}$ we get $\vdash_{L} A\left[B_{0} / p_{0}, \ldots, B_{n} / p_{n}\right]$. In particular, for any $f \in \operatorname{Prop}^{\omega}$, we have $\vdash_{L} A^{f}$. As a regular $L$-theory contains all $L$-theorems, this implies that for all $a \in 0_{L}, A^{f} \in a$, hence $\mathcal{M}_{L}, a, f \models A$ by the Truth Lemma. Thus $A$ is valid in $\mathcal{M}_{L}$ as required.

Theorem 4.11. If $L$ is an inductively defined logic, then $\mathcal{M}_{L} \models A$ implies $\vdash_{L} A$.

Proof. Suppose $\nvdash_{L} A$, and choose $n$ such that the free variables of $A$ are among $p_{0}, \ldots, p_{n}$. Choose distinct constants $c_{0} \ldots c_{n}$ not occurring in $A$. Then by the rule Sub of Lemma 2.2, $\nvdash_{L} A\left[c_{0} / p_{0}, \ldots, c_{n} / p_{n}\right]$. Hence by Corollary 4.2 there is some regular prime L-theory $\Gamma$ such that $A\left[c_{0} / p_{0} \ldots c_{n} / p_{n}\right] \notin \Gamma$, so $\Gamma \notin\left\|A\left[c_{0} / p_{0} \ldots c_{n} / p_{n}\right]\right\|_{L}$.

Now take any $f \in \operatorname{Prop}_{L}^{\omega}$ such that $f(i)=\left\|c_{i}\right\|_{L}$ for all $0 \leq i \leq n$. Then by Lemma 4.6, $\left\|A^{f}\right\|_{L}=\left\|A\left[c_{0} / p_{0}, \ldots, c_{n} / p_{n}\right]\right\|_{L}$. Hence $\Gamma \notin\left\|A^{f}\right\|_{L}$. But $\left\|A^{f}\right\|_{L}=|A|^{\mathcal{M}_{L}} f$ by the Truth Lemma 4.9, so $\mathcal{M}_{L}, \Gamma, f \not \models A$. Since $\Gamma \in 0_{L}$, this shows that $A$ is not valid in $\mathcal{M}_{L}$. 


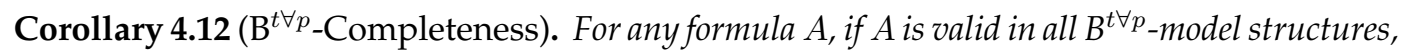
then $A$ is a $B^{t \forall p}$-theorem.

Proof. If $A$ is valid in all $\mathrm{B}^{t \forall p}$-model structures, then it is valid in $\mathcal{M}_{L}$ where $L$ is the inductively defined logic $\mathrm{B}^{t \forall p}$.

\section{Completeness for Inductively Defined Logics}

An inductively defined logic is one specified by adding to the axiomatisation of $\mathrm{B}^{t \forall p}$ some set $\Sigma$ of axioms from the long list of optional axioms given at the end of Section 2 . Now we give, for each axiom from that list, a corresponding condition on model structures. We use the following definitions:

$R^{2} a b c d$ iff there is some $x \in K$ such that (Rabx and $R x c d$ )

$R^{2} a(b c) d$ iff there is some $x \in K$ such that (Rbcx and $R a x d$ )

$R^{3} a b(c d) e$ iff there is some $x \in K$ such that $\left(R^{2} a b x e\right.$ and $\left.R c d x\right)$.

(CB1) Raaa

(CB2) Rabc implies $R^{2} a(a b) c$

(CB3) $\quad R^{2} a b c d$ implies $R^{2} b(a c) d$

(CB4) $\quad R^{2} a b c d$ implies $R^{2} a(b c) d$

(CB5) Rabc implies $R^{2} a b b c$

(CB6) Rabc implies Rbac

(CB7) $\quad R^{2} a b c d$ implies $R^{2} a c b d$

(CB8) $\quad R^{2}$ abcd implies $R^{3} a c(b c) d$

(CB9) $\quad R^{2}$ abcd implies $R^{3} b c(a c) d$

(CB10) Rabc implies $b \leq c$

(CB11) Rabc implies $a \leq c$

(CB12) $\quad R^{2} a b c d$ implies $a \leq d$

(CB13) Rabc implies $(a \leq c$ and $b \leq c)$

(CB14) $\quad R^{2} a b c d$ implies (Racd and $R b c d$ )

(CB15) $R^{2} a b c d$ implies there is some $x$ such that $b \leq x, c \leq x$ and Raxd

(CB16) $\quad(a \leq b$ and $x \in 0)$ implies $a \leq x$

(CB17) $a \leq b$ or $b \leq a$

(CB18) Rabc implies $(a \leq c$ or $b \leq c)$

(CB19) Rabc implies (Rbac and $a \leq c$ )

(CB20) (Rabc and Rade) implies there is some $x$ such that $(b \leq x$ and $d \leq x$ and (Raxc or $\operatorname{Raxe}))$

(CC1) $x \in 0$ implies Raxa

(CC2) $\quad x \in 0$ implies $R x x x$

(CC3) for any $x \in 0, R^{2} x b c d$ implies $R^{2} b(x c) d$

(CC4) for any $x \in 0, x^{*} \leq x$

(CC5) for any $x \in 0$, Raxc implies $a \leq c$

(CD1) (Rabc and Rade) implies there is some $x$ such that $\left(b \leq x\right.$ and $c^{*} \leq x$ and $\left.R a x b^{*}\right)$

(CD2) for any $x \in 0, x^{*} \leq x$

(CD3) $R a a^{*} a$

(CD4) Rabc implies $R a c^{*} b^{*}$

(CD5) $a^{*} \leq a$

(CD6) Rabc implies $a \leq b^{*}$

(CD7) (Rabc and $\left.R a^{*} d e\right)$ implies $(d \leq c$ or $b \leq e)$

(CD8) Rabc implies there is some $x$ such that $\left(R a c^{*} x\right.$ and for any $d, e$ (Rx $x^{*} d e$ implies $\left.\left.d \leq b^{*}\right)\right)$ 
(CE1) $\quad \forall_{n}(\varphi \Rightarrow \psi) f \subseteq\left(\forall_{n} \varphi \Rightarrow \forall_{n} \psi\right) f$

$(\mathrm{CE} 2) \quad \prod_{P \in \operatorname{Prop}}(\varphi f \cup \psi f[P / n]) \subseteq\left(\varphi \cup \forall_{n} \psi\right) f$

(CE3) $\quad\left(\forall_{n}\left(\varphi_{n} \Rightarrow \varphi_{n}\right) \Rightarrow \psi\right) f \subseteq \psi f$

The conditions other that (CE1)-(CE3) are those given in [11, pp. 300-301, 352] for dealing with the non-quantified axioms.

Fix any inductively defined logic $L=L_{\Sigma}$. An $L$-model structure is a $\mathrm{B}^{t \forall p}$-model structure that satisfies all of the above conditions corresponding to the members of $\Sigma$. An $L$-model is a $\mathrm{B}^{t \forall p}$-model on an $L$-model structure. We will show that $L$ is characterised by validity in $L$-model structures.

Theorem 5.1 (Soundness). Every L-theorem is valid in all L-model structures.

Proof. It is shown in [11] that all of our non-quantificational optional axioms are valid in all basic model structures that satisfy their corresponding conditions. We show the same here for the quantification axioms E1-E3.

Consider an arbitrary L-model $\mathcal{M}$. We repeatedly use the result $\varphi_{A}^{\mathcal{M}} f=|A|^{\mathcal{M}} f$ of Lemma 3.2, together with the inductive definitions of $\varphi_{A}^{\mathcal{M}} f$ and $|A|^{\mathcal{M}} f$.

(E1) Suppose CE1 holds. Let $f \in$ Prop $^{\omega}$. Then using Lemma 3.2 etc. we have

$$
\begin{aligned}
\left|\forall p_{n}(A \rightarrow B)\right|^{\mathcal{M}} f & =\varphi_{\forall p_{n}(A \rightarrow B)}^{\mathcal{M}} f \\
& =\forall_{n}\left(\varphi_{A}^{\mathcal{M}} \Rightarrow \varphi_{B}^{\mathcal{M}}\right) f \\
& \subseteq\left(\forall_{n} \varphi_{A}^{\mathcal{M}} \Rightarrow \forall_{n} \varphi_{B}^{\mathcal{M}}\right) f \quad \text { by CE1 } \\
& =\left|\forall p_{n} A \rightarrow \forall p_{n} B\right|^{\mathcal{M}} f .
\end{aligned}
$$

Hence $\mathcal{M} \models \forall p_{n}(A \rightarrow B) \rightarrow\left(\forall p_{n} A \rightarrow \forall p_{n} B\right)$ by the Semantic Entailment Lemma 3.4.

(E2) Suppose CE2 holds, and $p_{n}$ is not free in $A$. Then

$$
\begin{array}{rlrl}
\left|\forall p_{n}(A \vee B)\right|^{\mathcal{M}} f & =\prod_{P \in \text { Prop }}\left(|A|^{\mathcal{M}} f[P / n] \cup|B|^{\mathcal{M}} f[P / n]\right) & \\
& =\prod_{P \in \text { Prop }}\left(|A|^{\mathcal{M}} f \cup|B|^{\mathcal{M}} f[P / n]\right) & & \text { by Lemma 3.5 } \\
& =\prod_{P \in \text { Prop }}\left(\varphi_{A}^{\mathcal{M}} f \cup \varphi_{B}^{\mathcal{M}} f[P / n]\right) & \\
& \subseteq\left(\varphi_{A}^{\mathcal{M} \cup \forall} \forall_{n}^{\mathcal{M}}\right) f & & \text { by CE2 } \\
& =\left|A \vee \forall p_{n} B\right|^{\mathcal{M}} f . &
\end{array}
$$

Hence $\mathcal{M} \models \forall p_{n}(A \vee B) \rightarrow\left(A \vee \forall p_{n} B\right)$ by Semantic Entailment.

(E3) Suppose (CE3) holds. Then

$$
\begin{aligned}
\left.\mid \forall p_{n}\left(p_{n} \rightarrow p_{n}\right) \rightarrow A\right)\left.\right|^{\mathcal{M}} f & =\left(\forall_{n}\left(\varphi_{n} \Rightarrow \varphi_{n}\right) \Rightarrow \varphi_{A}^{\mathcal{M}}\right) f \\
& \subseteq \varphi_{A}^{\mathcal{M}} f=|A|^{\mathcal{M}} f \quad \text { by CE3 }
\end{aligned}
$$

so $\mathcal{M} \models\left(\forall p_{n}\left(p_{n} \rightarrow p_{n}\right) \rightarrow A\right) \rightarrow A$ by Semantic Entailment.

Lemma 5.2. $\mathcal{M}_{L}$ is an L-model structure.

Proof. It has to be shown that $\mathcal{K}_{L}$ is an L-model structure. We prove here the cases for the conditions CE1-CE3, and refer the reader to Chapters 4 and 5 of [11] for the cases of the conditions corresponding to other possible axioms of $L$. Take any $\varphi, \psi \in \operatorname{PropFun}_{L}$, so by definition $\varphi=\varphi_{L}^{A}$ and $\psi=\varphi_{L}^{B}$ for some formulas $A$ and $B$. We make repeated use of the equations of (4.1) and Lemma 4.8, along with (4.3) and the definition of $\varphi_{L}^{A} f$ as $\left\|A^{f}\right\|_{L}$.

(CE1) We have

$$
\forall_{n}\left(\varphi_{L}^{A} \Rightarrow \varphi_{L}^{B}\right) f=\left(\varphi_{L}^{\forall p_{n}(A \rightarrow B)}\right) f=\left\|\forall p_{n}(A \rightarrow B)^{f}\right\|_{L}=\left\|\forall p_{n}\left(A^{f \backslash n} \rightarrow B^{f \backslash n}\right)\right\|_{L} .
$$


But $\left\|\forall p_{n}\left(A^{f \backslash n} \rightarrow B^{f \backslash n}\right)\right\|_{L} \subseteq\left\|\forall p_{n}\left(A^{f \backslash n}\right) \rightarrow \forall p_{n}\left(B^{f \backslash n}\right)\right\|_{L}$ by axiom E1 and Lemma 4.3. Since

$$
\left\|\forall p_{n}\left(A^{f \backslash n}\right) \rightarrow \forall p_{n}\left(B^{f \backslash n}\right)\right\|_{L}=\left\|\left(\forall p_{n} A\right)^{f} \rightarrow\left(\forall p_{n} B\right)^{f}\right\|_{L}=\left(\forall_{n} \varphi_{L}^{A} \Rightarrow \forall_{n} \varphi_{L}^{B}\right) f,
$$

this proves $\forall_{n}\left(\varphi_{L}^{A} \Rightarrow \varphi_{L}^{B}\right) f \subseteq\left(\forall_{n} \varphi_{L}^{A} \Rightarrow \forall_{n} \varphi_{L}^{B}\right) f$, giving CE1 for $\mathcal{K}_{L}$.

(CE2) Fix an $f \in$ Prop $^{\omega}$ and, to avoid confusion of value-assignments, write $A^{\prime}$ for the closed formula $A^{f}$. Then for any $P \in \operatorname{Prop}$,

$$
\varphi_{L}^{A} f \cup \varphi_{L}^{B} f[P / n]=\left\|A^{\prime} \vee\left(B^{f[P / n]}\right)\right\|_{L} .
$$

But by (4.2) and the fact that $A^{\prime}$ is closed and hence unchanged by substitution,

$$
A^{\prime} \vee\left(B^{f[P / n]}\right)=A^{\prime} \vee\left(B^{f \backslash n}\left[P / p_{n}\right]\right)=\left(A^{\prime} \vee B\right)^{f \backslash n}\left[P / p_{n}\right]
$$

Hence

$$
\prod_{P \in \operatorname{Prop}_{L}}\left(\varphi_{L}^{A} f \cup \varphi_{L}^{B} f[P / n]\right)=\prod_{P \in \operatorname{Prop}_{L}}\left\|\left(A^{\prime} \vee B\right)^{f \backslash n}\left[P / p_{n}\right]\right\|_{L}=\left\|\forall p_{n}\left(\left(A^{\prime} \vee B\right)^{f \backslash n}\right)\right\|_{L}
$$

by Lemma 4.7. But by axiom E2, as $p_{n}$ is not free in $A^{\prime}$,

$$
\left\|\forall p_{n}\left(\left(A^{\prime} \vee B\right)^{f \backslash n}\right)\right\|_{L}=\left\|\forall p_{n}\left(A^{\prime} \vee\left(B^{f \backslash n}\right)\right)\right\|_{L} \subseteq\left\|A^{\prime} \vee \forall p_{n}\left(B^{f \backslash n}\right)\right\|_{L} .
$$

Since $\left\|A^{\prime} \vee \forall p_{n}\left(B^{f \backslash n}\right)\right\|_{L}=\varphi_{L}^{A} f \cup\left\|\left(\forall p_{n} B\right)^{f}\right\|_{L}$ (by (4.3)) $=\varphi_{L}^{A} f \cup \varphi_{L}^{\forall p_{n} B} f$, this all leads to

$$
\prod_{P \in \operatorname{Prop}_{L}}\left(\varphi_{L}^{A} f \cup \varphi_{L}^{B} f[P / n]\right) \subseteq\left(\varphi_{L}^{A} \cup \forall_{n} \varphi_{L}^{B}\right) f
$$

establishing CE2 for $\mathcal{K}_{L}$.

(CE3) Since $\varphi_{n}=\varphi_{L}^{p_{n}}$ in $\mathcal{K}_{L}$,

$$
\left(\forall_{n}\left(\varphi_{n} \Rightarrow \varphi_{n}\right) \Rightarrow \varphi_{L}^{A}\right) f=\left\|\left(\forall p_{n}\left(p_{n} \rightarrow p_{n}\right)^{f} \rightarrow A^{f}\right)\right\|_{L}=\left\|\left(\forall p_{n}\left(p_{n} \rightarrow p_{n}\right) \rightarrow A^{f}\right)\right\|_{L}
$$

as $\forall p_{n}\left(p_{n} \rightarrow p_{n}\right)$ is closed. But using axiom E3,

$$
\left\|\left(\forall p_{n}\left(p_{n} \rightarrow p_{n}\right) \rightarrow A^{f}\right)\right\|_{L} \subseteq\left\|A^{f}\right\|_{L}=\varphi_{L}^{A} f,
$$

so CE3 follows for $\mathcal{K}_{L}$.

Corollary 5.3 (Completeness). For any inductively defined logic $L$, if $A$ is valid in all $L$-model structures, then $A$ is an L-theorem.

Proof. By Lemma 5.2 and Theorem 4.11.

\section{Incompleteness}

A model structure or model is called full if its set Prop of admissible propositions contains every one of its hereditary subsets. In that case, if $S \subseteq \operatorname{Prop}$ then $\bigcap S$ is admissible, being hereditary, and so $\prod S=\bigcap S$. It follows that in any full model,

$$
\left|\forall p_{n} A\right|^{\mathcal{M}} f=\bigcap_{P \in \text { Prop }}|A|^{\mathcal{M}} f[P / n],
$$

and so universal quantifiers have the standard semantics

$$
\mathcal{M}, a, f \models \forall p_{n} A \text { iff } \mathcal{M}, a, f[P / n] \models A \text { for all } P \in \text { Prop. }
$$


Routley and Meyer speculated in [10, p. 235] that the system RP is incomplete for its full model-structures, i.e. that there are formulas valid in all full RP-model structures that are not RP-theorems. This was confirmed by Kremer in [7] by proving that the set of all formulas valid in all full RP-model structures is not recursively axiomatisable, and indeed is recursively isomorphic to full second-order logic. This shows that the use we have made of models with a restricted set of admissible propositions is essential for providing a complete relational semantics for RP.

But what of other logics, such as EP? In this final section we show that there are numerous inductively defined logics that are incomplete for their full model-structures. To state our results most generally, let $L_{A l g}$ be the smallest logic that contains all of the axiom schemes

$$
\text { A1-A9, B1-B5, B8-B10, B14, B18, C1-C4 and D1-D5. }
$$

We will define a particular formula $I n c$ such that

(1) Inc is valid in all full model structures whatsoever; and

(2) Inc is not a theorem of $L_{A l g}$.

It follows that every sublogic $L$ of $L_{A l g}$ is incomplete for its full model-structures, since Inc is valid in all full $L$-models by (1), but is not a theorem of $L$ by (2). In particular, it can be shown that $E P$ is a sublogic of $L_{A l g}$, as is $\mathrm{EM}^{t \forall p}$, the extension of EP by $t$ and the mingle axiom $(A \rightarrow B) \rightarrow((A \rightarrow B) \rightarrow(A \rightarrow B))$, so the incompleteness applies to these logics, and to all of their sublogics.

Now to define Inc, let Exm (for Excluded Middle) be the sentence $\forall p(p \vee \neg p)$, and let Atm (for Atom) be the sentence

$$
\exists q(q \wedge \forall r(q \rightarrow r \vee q \rightarrow \neg r)) .
$$

Then Inc is the sentence $\operatorname{Exm} \wedge t \rightarrow$ Atm.

After showing (1) we will show (2) by defining a semantics, using Boolean algebras, in which Atm asserts of an algebra that it contains an atom (in a sense: see Lemma 6.5). Bull [5] used a similar atomicity assertion to show the incompleteness of the propositionally quantified modal logic S5 for its full models.

The main fact about full models that we need, in addition to their standard semantics for quantifiers, is that in a full model the hereditary sets $[a)=\{b: a \leq b\}$ generated by each point are admissible.

Lemma 6.1. In any full model $\mathcal{M}$, if $x \in \mid$ Exm $\left.\right|^{\mathcal{M}}$ then $x^{*} \leq x$.

Proof. Note that as Exm is a sentence, the value $|E x m|^{\mathcal{M}} f$ is independent of $f$ by Lemma 3.5, so the notation $|E x m|^{\mathcal{M}}$ is justified. Using (6.1) we see that

$$
|E x m|^{\mathcal{M}}=\bigcap_{P \in \text { Prop }}(P \cup-P) .
$$

Suppose $x \in|E x m|^{\mathcal{M}}$. As $\mathcal{M}$ is full, the hereditary set $\left[x^{*}\right)=\left\{a: x^{*} \leq a\right\}$ is in Prop and therefore $x \in\left[x^{*}\right) \cup-\left[x^{*}\right)$. But if $x \in-\left[x^{*}\right)$, then $x^{*} \notin\left[x^{*}\right)$ by the definition of $-\left[x^{*}\right)$, contradicting the reflexivity of $\leq(\mathrm{P} 2)$. So it must be that $x \notin-\left[x^{*}\right)$ and hence $x \in\left[x^{*}\right)$, i.e. $x^{*} \leq x$.

To analyse the sentence $A t m$ we first describe the semantics of the defined notion $\exists p_{n} A=d f$ $\neg\left(\forall p_{n}(\neg A)\right)$ when interpreted in full models.

Lemma 6.2. For any full model $\mathcal{M}$ and $f \in$ Prop $^{\omega}$,

$$
\left|\exists p_{n} A\right|^{\mathcal{M}} f=\bigcup_{P \in \text { Prop }}|A|^{\mathcal{M}} f[P / n],
$$

so existential quantifiers have the standard semantics

$$
\mathcal{M}, a, f \models \exists p_{n} A \text { iff } \mathcal{M}, a, f[P / n] \models A \text { for some } P \in \text { Prop. }
$$


Proof. $\left|\exists p_{n} A\right|^{\mathcal{M}} f=-\bigcap_{P \in \text { Prop }}-|A|^{\mathcal{M}} f[P / n]$, so $a \in\left|\exists p_{n} A\right|^{\mathcal{M}}$ iff $a^{*} \notin \bigcap_{P \in \text { Prop }}-|A|^{\mathcal{M}} f[P / n]$ iff there is some $P \in$ Prop such that $a^{*} \notin-|A|^{\mathcal{M}} f[P / n]$. But $a^{*} \notin-|A|^{\mathcal{M}} f[P / n]$ iff $a=$ $a^{* *} \in|A|^{\mathcal{M}} f[P / n]$.

Lemma 6.3. In any full model $\mathcal{M}$, if $x^{*} \leq x \in 0$, then $x \in \mid$ Atm $\left.\right|^{\mathcal{M}}$.

Proof. Suppose $x^{*} \leq x \in 0$. Using (6.1) and (6.2) we see that

$$
|A t m|^{\mathcal{M}}=\bigcup_{Q \in \text { Prop }}\left(Q \cap \bigcap_{R \in \text { Prop }}((Q \Rightarrow R) \cup(Q \Rightarrow-R)) .\right.
$$

Now let $Q=[x)=\{a: x \leq a\}$. Then $x \in Q \in$ Prop, as $\mathcal{M}$ is full. Thus it suffices to show

$$
x \in \bigcap_{R \in \text { Prop }}((Q \Rightarrow R) \cup(Q \Rightarrow-R))
$$

to conclude from this that $x \in \mid$ Atm $\left.\right|^{\mathcal{M}}$.

So take any $R \in \operatorname{Prop}$ and suppose $x \notin([x) \Rightarrow R)$. Then there must be some $a, b \in K$ such that Rxab, $a \in[x)$ and $b \notin R$. So we know that $x^{*} \leq x, x \leq a$, and $a \leq b$ as $x \in 0$. Hence $b^{*} \leq a^{*} \leq x^{*} \leq x$ by (P5), hence $b^{*} \leq x$ by transitivity (P2). Given $b^{* *}=b \notin R$ it follows that $b^{*} \in-R$, and as $-R$ is hereditary $(R \in$ Prop and Prop closed under - ) and $b^{*} \leq x$ we have that $x \in-R$. Now to show $x \in([x) \Rightarrow-R)$ take any $c, d \in K$ and suppose $R x c d$ and $c \in[x)$. Then $x \leq c$ and $c \leq d$, hence $x \leq d$. So as $x \in-R$ it follows that $d \in-R$. Therefore $x \in([x) \Rightarrow-R)$.

Putting all this together, we see that for any $R \in$ Prop, either $x \in([x) \Rightarrow R)$ or $x \in$ $([x) \Rightarrow-R)$, so

$$
\left.x \in \bigcap_{R \in \text { Prop }}([x) \Rightarrow R) \cup([x) \Rightarrow-R)\right)
$$

as required.

Theorem 6.4. Every full model validates the sentence Exm $\wedge t \rightarrow$ Atm.

Proof. Let $\mathcal{M}$ be full and $x \in|E x m|^{\mathcal{M}} \cap|t|^{\mathcal{M}}$. Then $x^{*} \leq x$ by Lemma 6.1, and $x \in 0$, so $x \in|A t m|^{\mathcal{M}}$ by Lemma 6.3. Hence $\mathcal{M} \models E x m \wedge t \rightarrow$ Atm by the Semantic Entailment Lemma 3.4.

To show that Exm $\wedge t \rightarrow$ Atm is not a theorem of $L_{A l g}$ we turn to algebraic semantics, using Boolean algebras

$$
\mathscr{B}=\left\langle\mathcal{B}, \wedge_{\mathscr{B}}, \vee_{\mathscr{B}},-\mathscr{B}, 0_{\mathscr{B}}, 1_{\mathscr{B}}\right\rangle
$$

that are complete in the sense of having a meet $\bigwedge \mathcal{S}$ and join $\bigvee \mathcal{S}$ of every subset $\mathcal{S} \subseteq \mathcal{B}$. On such a Boolean algebra we define an binary operation $\Rightarrow$ by

$$
a \Rightarrow b= \begin{cases}1_{\mathscr{B}} & \text { if } a \leq b, \\ 0_{\mathscr{B}} & \text { otherwise }\end{cases}
$$

This will be used to interpret the connective $\rightarrow$.

A Boolean algebraic model has the form $\mathscr{M}=\langle\mathscr{B}, V\rangle$, where $\mathscr{B}$ is a complete Boolean algebra and $V$ is a function $V: C o n \rightarrow \mathscr{B}$ providing a valuation of the constants of our propositional language. In such a model, for each function $f: \omega \rightarrow \mathscr{B}$, acting as a variableassigment, we inductively define a value $|A|^{\mathscr{M}} f$ in $\mathcal{B}$ for each formula $A$. The base of the induction is given by $|c|^{\mathscr{M}} f=V(c),\left|p_{n}\right|^{\mathscr{M}} f=f(n)$ and $|t|^{\mathscr{M}} f=1_{\mathscr{B}}$. The connectives $\rightarrow, \wedge, \neg$ are inductively interpreted by the operations $\Rightarrow, \wedge_{\mathscr{B}},-\mathscr{B}$, and the quantifier case is given by

$$
\left|\forall p_{n} A\right|^{\mathscr{M}} f=\bigwedge_{b \in \mathcal{B}}|A|^{\mathscr{M}} f[b / n]
$$


Then we get $|A \vee B|^{\mathscr{M}} f=|A|^{\mathscr{M}} f \vee_{\mathscr{B}}|B|^{\mathscr{M}} f$ and

$$
\left|\exists p_{n} A\right|^{\mathscr{M}} f=\bigvee_{b \in \mathcal{B}}|A|^{\mathscr{M}} f[b / n],
$$

as usual for Boolean algebraic semantics. A formula A is valid in $\mathscr{M}$ if $|A|^{\mathscr{M}} f=1_{\mathscr{B}}$ for every $f \in \mathscr{B}^{\omega}$.

It can be shown that every theorem of $L_{A l g}$ is valid in every Boolean algebraic model. All of the axioms

$$
\text { A1-A10, B1-B5, B8-B10, B14, B18, C1-C4 and D1-D5 }
$$

are valid, and all the rules R1-R7, RIC, and E1-E3 preserves that validity. Showing this involves a great deal of fairly routine algebraic reasoning which is left to the interested reader. Proof of validity of the quantifier axioms makes use of analogues of Lemmas 3.5 and 3.6, namely:

- If $f$ and $g$ agree on the free variables of $A$, then $|A|^{\mathscr{M}} f=|A|^{\mathscr{M}} g$.

- If $p_{n}$ is free for $B$ in $A$, then $\left|A\left[B / p_{n}\right]\right|^{\mathcal{M}} f=|A|^{\mathcal{M}} f\left[|B|^{\mathcal{M}} f / n\right]$.

These algebraic models do not validate such schemes as B6, B7 and C5, so our incompleteness method does not apply to RQ. But that logic was dealt with by Kremer's result.

We now show that there are Boolean algebraic models invalidating Inc. Recall that an atom of a Boolean algebra is a non-zero element $a$ such that if $b \leq a$, then $b=a$ or $b=0$. Equivalently, a non-zero $a$ is an atom iff $a \leq b$ or $a \leq-b$ for all $b$. Note that as Atm is a sentence with no constants, it has a fixed value in any model that is independent of $V$, and so can be denoted $|A t m|^{\mathscr{B}}$.

Lemma 6.5. If $\mid$ Atm $\left.\right|^{\mathscr{B}} \neq 0$, then $\mathscr{B}$ has an atom.

Proof. Suppose $|A t m|^{\mathscr{B}} \neq 0$. Then as

$$
|A t m|^{\mathscr{B}}=\bigvee_{a \in \mathcal{B}}\left(a \wedge_{\mathscr{B}} \bigwedge_{b \in \mathcal{B}}\left(a \Rightarrow b \vee_{\mathscr{B}} a \Rightarrow-b\right)\right),
$$

there must be some $a \in \mathcal{B}$ with

$$
a \wedge_{\mathscr{B}} \bigwedge_{b \in \mathcal{B}}\left(a \Rightarrow b \vee_{\mathscr{B}} a \Rightarrow-b\right) \neq 0 .
$$

Hence $a \neq 0$, and for every $b,\left(a \Rightarrow b \vee_{\mathscr{B}} a \Rightarrow-b\right) \neq 0$, so either $a \Rightarrow b \neq 0$ or $a \Rightarrow-b \neq 0$, hence $a \leq b$ or $a \leq-b$. Thus $a$ is an atom.

Corollary 6.6. If $\mathscr{B}$ is a complete and atomless Boolean algebra, then Exm $\wedge t \rightarrow$ Atm is not valid in $\mathscr{B}$.

Proof. By the Lemma, $\mid$ Atm $\left.\right|^{\mathscr{B}}=0$. But

$$
\mid \operatorname{Exm} \wedge t^{\mathscr{B}}=\left(\bigwedge_{a \in \mathcal{B}}\left(a \vee_{\mathscr{B}}-a\right)\right) \wedge_{\mathscr{B}} 1=1,
$$

So $|\operatorname{Exm} \wedge t \rightarrow A t m|^{\mathscr{B}}=1 \Rightarrow 0=0$.

Since there do exist complete atomless Boolean algebras - for instance the algebra of regular open subsets of the real line - it follows that there are algebraic models that validate $L_{A l g}$ but do not validate Inc. So Inc is not an $L_{A l g}$-theorem, which gives our overall incompleteness result. 


\section{References}

[1] Alan Ross Anderson. An intensional interpretation of truth-values. Mind, 81:348-371, 1972.

[2] Alan Ross Anderson and Nuel D. Belnap, Jr. Enthymemes. J. Philosophy, 58(23):713723, 1961.

[3] Alan Ross Anderson, Nuel D. Belnap, Jr., and J. Michael Dunn. Entailment. The logic of relevance and necessity. Vol. II. Princeton University Press, Princeton, NJ, 1992.

[4] Katalin Bimbó and J. Michael Dunn. Relational semantics for Kleene Logic and Action Logic. Notre Dame Journal of Formal Logic, 46(4):461-490, 2005.

[5] R. A. Bull. On modal logic with propositional quantifiers. J. Symbolic Logic, 34(2):257263, 1969.

[6] Leon Henkin. Completeness in the theory of types. J. Symbolic Logic, 15:81-91, 1950.

[7] Philip Kremer. Quantifying over propositions in relevance logic: nonaxiomatisability of primary interpretations of $\forall p$ and $\exists p$. J. Symbolic Logic, 58(1):334-349, 1993.

[8] Edwin D. Mares and Robert Goldblatt. An alternative semantics for quantified relevant logic. J. Symbolic Logic, 71(1):163-187, 2006.

[9] Robert K. Meyer. Intuitionism, entailment, negation. In Hughes Leblanc, editor, Truth, Syntax and Modality, pages 168-198. North-Holland, Amsterdam, 1973.

[10] Richard Routley and Robert K. Meyer. The semantics of entailment. In Hughes Leblanc, editor, Truth, Syntax and Modality, pages 199-243. North-Holland, Amsterdam, 1973.

[11] Richard Routley, Val Plumwood, Robert K. Meyer, and Ross T. Brady. Relevant logics and their rivals. Part I. Ridgeview Publishing Co., Atascadero, CA, 1982. 